\title{
Exotic quarkonium states in CMS
}

\author{
Leonardo Cristella ${ }^{1,2, a}$, on behalf of the CMS Collaboration \\ ${ }^{1}$ Universitá degli Studi di Bari \\ ${ }^{2}$ INFN - Sezione di Bari
}

\begin{abstract}
The studies of the production of the $X(3872)$, either prompt or from B hadron decays, and of the $J / \psi \phi$ mass spectrum in B hadron decays have been carried out by using $p p$ collisions at $\sqrt{s}=7 \mathrm{TeV}$ collected with the CMS detector at the LHC. The cross-section ratio of the $X(3872)$ with respect to the $\psi(2 S)$ in the $J / \psi \pi^{+} \pi^{-}$decay channel and the fraction of $X(3872)$ coming from B-hadron decays are measured as a function of transverse momentum $\left(p_{\mathrm{T}}\right)$, covering unprecedentedly high values of $p_{\mathrm{T}}$. For the first time, the prompt production cross section for the $X(3872)$ times the unknown branching fraction for the decay of $X(3872) \rightarrow J / \psi \pi^{+} \pi^{-}$is extracted differentially in $p_{\mathrm{T}}$ and compared to theoretical predictions based on the Non-Relativistic QCD (NRQCD) factorization approach. The dipion invariant-mass spectrum of the $J / \psi \pi^{+} \pi^{-}$system in the $X(3872)$ decay is also investigated.

A peaking structure in the $J / \psi \phi$ mass spectrum near threshold is observed in $B^{ \pm} \rightarrow$ $J / \psi \phi K^{ \pm}$decays. The data sample, selected on the basis of the dimuon decay mode of the $J / \psi$, corresponds to an integrated luminosity of $5.2 \mathrm{fb}^{-1}$. Fitting the structure to an $\mathrm{S}$-wave relativistic Breit-Wigner lineshape above a three-body phase-space nonresonant component gives a signal statistical significance exceeding five standard deviations. The fitted mass and width values are $m=4148.0 \pm 2.4$ (stat.) \pm 6.3 (syst.) $\mathrm{MeV}$ and $\Gamma=$ $28_{-11}^{+15}$ (stat.) \pm 19 (syst.) $\mathrm{MeV}$, respectively. Evidence for an additional peaking structure at higher $J / \psi \phi$ mass is also reported.

The search for resonance-like structures in the $B_{s}^{0} \pi^{ \pm}$invariant mass spectrum do not show any unexpected result. An upper limit on the relative production of the claimed $X(5568)$ and $B_{s}$ multiplied by the unknown branching fraction of the decay $X(5568) \rightarrow B_{s} \pi^{ \pm}$is estimated to be $3.9 \%$ at $95 \% \mathrm{CL}$ in the most conservative case.
\end{abstract}

\section{Introduction}

The observation of many new states, with masses above the open-charm threshold, that do not fit into the conventional quark model has renewed the interest in exotic quarkonium spectroscopy. The $X(3872)$ was discovered by the Belle experiment in 2003 [1] and, despite a series of detailed studies performed at B-factories and Tevatron, its nature still remains unknown. Some interpretations describe the $X(3872)$ as a molecular state (loosely bound state of $D^{0 *} \bar{D}^{0}$ ) or as a tetraquark state (bound state of diquark-antidiquark) [2]. At Large Hadron Collider (LHC) there is the opportunity to study this state and to measure the prompt $X(3872)$ production rate as a function of transverse momentum. In

\footnotetext{
a e-mail: leonardo.cristella@ cern.ch
} 
this note, the measurement of production of the $X(3872)$ in $p p$ collisions at a centre-of-mass energy $\sqrt{s}=7 \mathrm{TeV}$ is presented in Section 2 .

The observation of $Y(3940)$ [10][11] near the $J / \psi \omega$ threshold motivates the search of other states close to the $J / \psi \phi$ threshold with similar characteristics explainable as threshold enhancements, rescattering effects or partner states with a new quarks' aggregate (tetraquark) or a molecular model $\left(D_{s}^{*} \bar{D}_{s}^{*}\right.$ partner of a $D^{*} \bar{D}^{*}$ loosely bound molecule). The CDF collaboration observed the $Y(4140)$ structure with a significance greater than five standard deviations [12], while LHCb collaboration initially did not confirm it and put an upper limit to its production [13] differently from the recent study in Ref.[14]. In Section 3, the $Y(4140)$ analysis performed by CMS is presented [3].

The evidence of a new $B_{s}^{0} \pi^{ \pm}$state presented by the $\mathrm{D} \varnothing$ Collaboration [16] raised a lot of interest within the community that studies exotic hadrons [17] and triggered this search at several hadron collider experiments, including CMS [18] and LHCb [19]. The CMS search for resonance-like structures in the $B_{s}^{0} \pi^{ \pm}$invariant mass spectrum has been performed using an integrated luminosity of $19.7 \mathrm{fb}^{-1}$ of pp collisions at $\sqrt{s}=8 \mathrm{TeV}$ as discussed in Section 4 .

\section{Measurements of the $X(3872)$ state}

The analysis is performed on the data recorded by the CMS experiment in 2011, corresponding to an integrated luminosity of $4.8 \mathrm{fb}^{-1}$. The $X(3872)$ is observed using the decays into $J / \psi \pi^{+} \pi^{-}$, with the subsequent decay of the $J / \psi$ into a pair of muons. This decay channel has a clean experimental signature in CMS thanks to the high granularity of the tracker and the high efficiency of $J / \psi$ identification. A detailed description of the detector can be found elsewhere [4]. The data are collected with requirements on the dimuon system imposed at the trigger level, such as rapidity within 1.25 and a transverse momentum threshold of $9.9 \mathrm{GeV}$ in order to benefit from a better mass resolution in the central rapidity region. The analysis is thus performed in the kinematic range of $p_{\mathrm{T}}$ of the $J / 4 \pi^{+} \pi^{-}$ system between 10 and $50 \mathrm{GeV}$ and the rapidity within $|y|<1.2$, collecting about $12000 X(3872)$ candidates. The event selection and the event simulations, used to determine acceptances and efficiencies, are described in detail in Ref.[5]. The $X(3872)$ is assumed to be an unpolarized state and its $J^{P C}$ is fixed to $1^{++}$as favoured by the existing studies [6].

\subsection{Measurement of the $X(3872)$ cross section ratio with respect to $\psi(2 S)$}

The ratio of the $X(3872)$ and $\psi(2 S)$ cross sections times their branching fraction to $J / \psi \pi^{+} \pi^{-}$is obtained from the measured numbers of signal events, $N_{X(3872)}$ and $N_{\psi(2 S)}$, correcting for the efficiency $(\epsilon)$ and acceptance $(A)$ estimated from simulations, according to

$$
\begin{aligned}
R & =\frac{\sigma(p p \rightarrow X(3872)+\text { anything }) \cdot B\left(X(3872) \rightarrow J / \psi \pi^{+} \pi^{-}\right)}{\sigma(p p \rightarrow \psi(2 S)+\text { anything }) \cdot B\left(\psi(2 S) \rightarrow J / \psi \pi^{+} \pi^{-}\right)} \\
& =\frac{N_{X(3872)} \cdot A_{\psi(2 S)} \cdot \epsilon_{\psi(2 S)}}{N_{\psi(2 S)} \cdot A_{X(3872)} \cdot \epsilon_{X(3872)}} .
\end{aligned}
$$

The signal yields, $N_{X(3872)}$ and $N_{\psi(2 S)}$, are determined from unbinned maximum-likelihood fits to the invariant-mass spectra of the $J / \psi \pi^{+} \pi^{-}$system, separately for the $X(3872)$ and $\psi(2 S)$ in different mass windows. The $\psi(2 S)$ is parametrized using two Gaussian functions with a common mean, while a single Gaussian is used for the $X(3872)$ signal and the nonresonant background is fitted with a second-order Chebyshev polynomial. The acceptances and efficiencies for the $X(3872)$ and $\psi(2 S)$ final states are determined from the simulation. Monte Carlo simulation studies are performed to determine the systematic uncertainties and a data-driven method is used to verify the efficiencies, 
as described in Ref.[5]. The main systematic uncertainty arises from the limited knowledge of the $X(3872)$ transverse momentum. Both $X(3872)$ and $\psi(2 S)$ states are considered unpolarized and no systematic uncertainty is assigned to cover other cases. The total uncertainty for each $p_{\mathrm{T}}$ interval is typically $10 \%$ statistical and $5-13 \%$ systematic. No significant dependence on $p_{\mathrm{T}}$ is observed.

\subsection{Measurement of the nonprompt fraction of $X(3872)$ production}

The fraction of $X(3872)$ produced from decays of B hadrons is named nonprompt fraction. The $X(3872)$ candidates from B hadron decays are selected based on the pseudo-proper decay lenght $\left(l_{x y}\right)$, defined in Ref.[5] and related to the distance between the primary vertex and the secondary one, formed by the $J / \psi \pi^{+} \pi^{-}$system. The data sample consists of events where an average of six $p p$ collisions in the same bunch crossing (pileup) occur and the selected primary vertex is the one that minimizes the distance from the secondary vertex along the beam direction. A B-hadron-enriched sample is obtained requiring $l_{x y}$ larger than $100 \mu \mathrm{m}$, with an estimated contribution from prompt $X(3872)$ below $0.1 \%$. The nonprompt fraction is then obtained from the ratio between the signal yields extracted from this B-hadron-enriched sample and the signal yields in the inclusive sample, after correction for the efficiencies of the decay-length-selection criteria determined from simulations. Detailed studies are performed to verify the stability of the results and to determine the systematic uncertainties leading to a total systematic uncertainty of $6-10 \%$, as described in Ref.[5]. The measurement is dominated by its statistical uncertainty $(\sim 20 \%)$. The $X(3872)$ nonprompt fraction reveals no significant dependence on transverse momentum. These results are obtained assuming null polarization for the $X(3872)$ and no systematic uncertainty is assigned.

\subsection{The prompt $X(3872)$ production cross section}

The cross section times branching fraction for prompt $X(3872)$ production is determined from the measurement of the cross section ratio $R$ and the nonprompt fraction, described above, combined with a previous result of the prompt $\psi(2 S)$ cross section obtained in CMS [7]. The latter measurement was performed using the $\psi(2 S) \rightarrow \mu^{+} \mu^{-}$decay mode and provides results as a function of transverse momentum up to $30 \mathrm{GeV}$, in the same rapidity range of this analysis. By means of this combination, the differential cross section for prompt $X(3872)$ production times the branching fraction is obtained as a function of $p_{\mathrm{T}}$, in the rapidity region $|y|<1.2$, as shown in Fig.1. The $X(3872)$ and $\psi(2 S)$ states are assumed to be unpolarized and no cancellation of systematic uncertainties is assumed in the combination. The main sources of systematic uncertainty are related to the measurement of $R$ and of the prompt $\psi(2 S)$ cross section [7]. The differential cross section for prompt $X(3872)$ production in $p p$ collisions at $\sqrt{s}=7 \mathrm{TeV}$ has also been predicted with a calculation made within the NRQCD factorization formalism [8]. In this calculation the cross section is expressed as the sum of parton cross sections for creating $c \bar{c}$ pairs with vanishing relative momentum multiplied by phenomenological constants and results are normalized using Tevatron measurements with the statistical uncertainty obtained from the experimental input data. The predictions from Ref.[8] were modified by the authors to match the phase-space of the measurement presented in this note. The comparison of this prediction with the data is shown in Fig. 1 and demonstrates that, while the shape is reasonably described, the predicted cross section is much larger than the measured one. The integrated prompt $X(3872)$ cross section times branching fraction for the kinematic region $10<p_{\mathrm{T}}<30 \mathrm{GeV}$ and $|y|<1.2$ is also determined to be

$$
\begin{gathered}
\sigma^{\text {prompt }}(p p \rightarrow X(3872)+\text { anything }) \cdot B\left(X(3872) \rightarrow J / \psi \pi^{+} \pi^{-}\right)= \\
1.06 \pm 0.11 \text { (stat. }) \pm 0.15(\text { syst. }) \text { nb. }
\end{gathered}
$$


This measured value is below the theoretical prediction for the prompt $X(3872)$ cross section times branching fraction in the same kinematic region, which is $4.01 \pm 0.88 \mathrm{nb}$ [8].

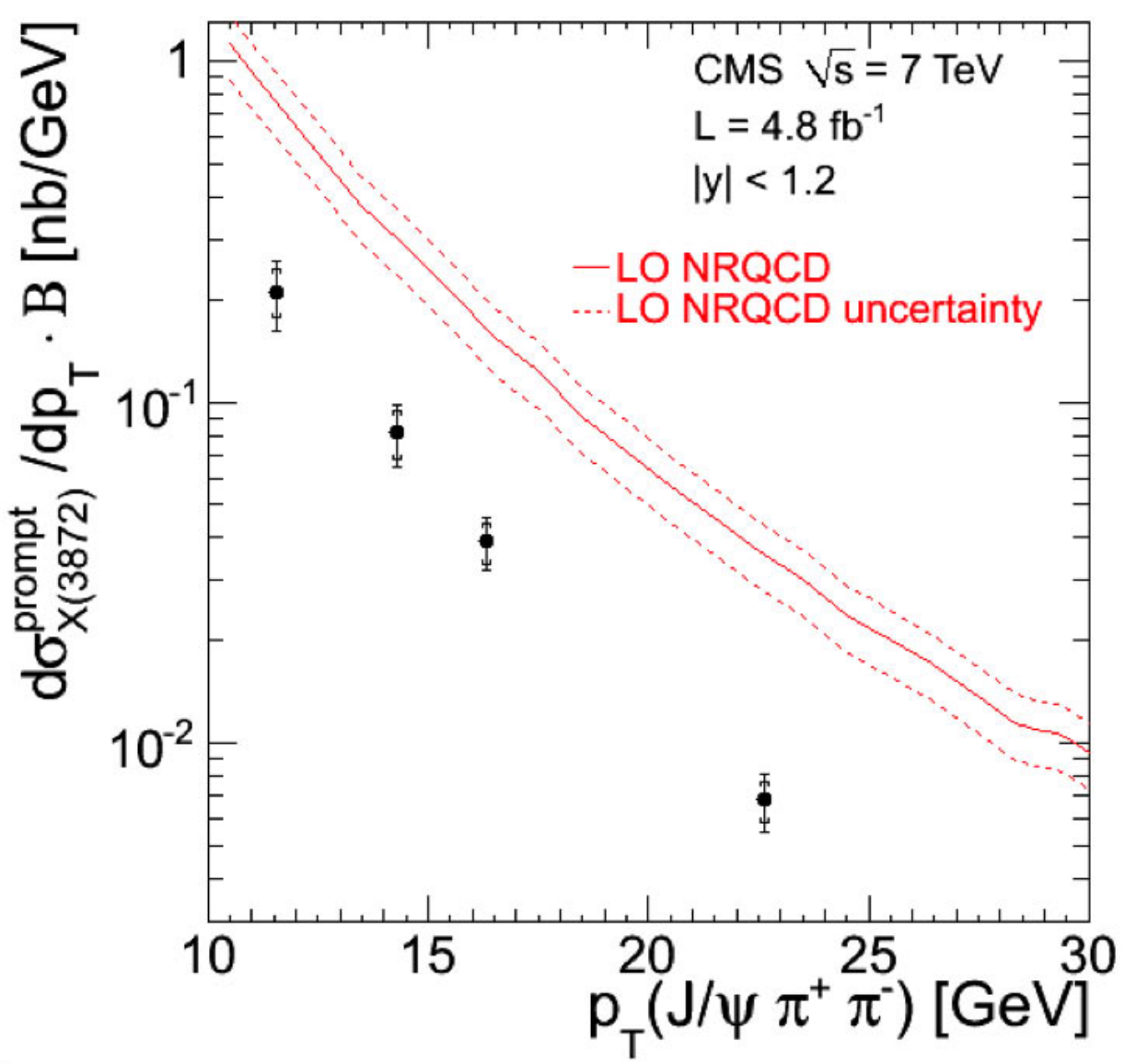

Figure 1. Measured differential cross section for prompt $X(3872)$ production times branching fraction of $X(3872) \rightarrow J / \psi \pi^{+} \pi^{-}$as a function of $p_{\mathrm{T}}$. The inner error bars indicate the statistical uncertainty while the outer error bars represent the total uncertainty. Theoretical prediction as explained in the text is shown by the solid line with the dotted lines representing the related uncertainty.

\subsection{Study of the $X(3872)$ decay properties}

The dipion invariant-mass distribution from $X(3872)$ decays to $J / \psi \pi^{+} \pi^{-}$is measured in order to investigate the decay properties of the $X(3872)$ and evaluate the presence of an intermediate $\rho^{0}$ state in the decay, as suggested by a previous study [9]. The measurement is made within the kinematic region $10<p_{\mathrm{T}}<50 \mathrm{GeV}$ and $|y|<1.25$. To extract the dipion invariant-mass spectrum from $X(3872)$ decays, the event sample is divided into intervals of $m\left(\pi^{+} \pi^{-}\right)$in the range $0.5-0.78 \mathrm{GeV}$. In each interval, a maximum-likelihood fit to the $J / \psi \pi^{+} \pi^{-}$invariant-mass distribution is performed with the signal modelled by a Gaussian. The position and width of the $X(3872)$ signal are fixed to the values 
obtained in the fit to the full sample. The dipion invariant-mass distribution is extracted from the signal yields obtained from these fits to the data in each interval, after correcting for detector acceptance and efficiency estimated from the simulation, as described in Ref.[5]. The main systematic uncertainties are related to the signal extraction $(10-20 \%)$ and to corrections due to acceptance and efficiency $(4-6 \%)$. The resulting dipion invariant-mass spectrum is normalized to the total cross section in the interval $0.5<m\left(\pi^{+} \pi^{-}\right)<0.78 \mathrm{GeV}$. The data are compared to $X(3872)$ signal simulations with and without an intermediate $\rho^{0}$ in the $X(3872)$ decay. The assumption of an intermediate $\rho^{0}$ decay gives better agreement with the data.

\section{Measurements of the $Y(4140)$ state}

\section{1 $Y(4140)$ decay reconstruction and signal extraction}

The $J / \psi$ candidates are recostructed by pairs of oppositely charged identified muons according to the selection implemented by the dimuon HLT, including muons' minimum $p_{\mathrm{T}}$ up to $4 \mathrm{GeV}, p_{\mathrm{T}}>7$ $\mathrm{GeV}$ and transverse flight length significance greater than 3. The $B^{ \pm} \rightarrow J / \psi \phi K^{ \pm}$candidates are recostructed by combining three additional good quality charged tracks, having $p_{\mathrm{T}}>1 \mathrm{GeV}$, pointing to the displaced $J / \psi$ vertex, with a total charge of \pm 1 and with kaon mass assigned (kaon track). The five tracks, with the $\mu^{+} \mu^{-}$system kinematically constrained to the $J / \psi$ mass nominal value, are required to form a good 3D vertex with $\chi^{2}$ probability greater than $1 \%$. The $K^{+} K^{-}$pair with lower mass must have a recostructed mass $1.008 \mathrm{GeV}<m\left(K^{+} K^{-}\right)<1.035 \mathrm{GeV}$ to be considered as a $\phi$ candidate. The selection criteria, designed to maximize the $B$ signal yield, were determined before examining the mass difference $\Delta m \equiv m\left(\mu^{+} \mu^{-} K^{+} K^{-}\right)-m\left(\mu^{+} \mu^{-}\right)$, that is the observable used to search for possibile structures in the $J / \psi \phi$ mass and investigated up to $1.568 \mathrm{GeV}$ in order to reject the reflection from $B_{s}^{0} \rightarrow \psi(2 S) \phi \rightarrow\left(J / \psi \pi^{+} \pi^{-}\right) \phi$.

\subsection{Background-subtracted and fit of the $\Delta m$ spectrum}

Signal Monte Carlo samples with specific values for $m(J / \psi \phi)$ (and thus for $\Delta m$ ) are produced to provide:

- $B$ mass resolution as a function of $J / \psi \phi$ mass values;

- absolute $B$ efficiency as a function of $J / \psi \phi$ mass values;

- $J / \psi \phi$ mass resolution as a function of $J / \psi \phi$ mass values.

The first information is needed by the used background subtraction method, the second by the relative efficiency correction and the third in the final $\Delta m$ fit.

The background subtraction is obtained by a bin-wise fit method. After dividing the $\Delta m$ spectrum in $20 \mathrm{MeV}$-sized 28 bins, the $B$ yield for each bin is extracted by a binned maximum likelihood fit to the corresponding $B$ candidates' mass spectrum. A second order Chebyshev polynomial is used for the background whereas the signal fit model consists of two gaussians with common mean (the nominal $B^{+}$mass) and with widths and their relative ratio fixed to $\Delta m$-dependent values predicted by the signal Monte Carlo. The resulting yields as a function of the binned $\Delta m$ provide the background-subtracted $\Delta m$ spectrum. This result is found to be consistent with the distribution that can be extracted by applying the alternative technique of sPlot [15].

After performing the background subtraction the resulting $\Delta m$ distribution and its $1 \mathrm{D}$-fit is shown in Fig. 2: the fit model, which accounts for the relative efficiency correction, includes the three-body phase-space profile for the background whereas each structure is modelled with an S-wave relativistic 


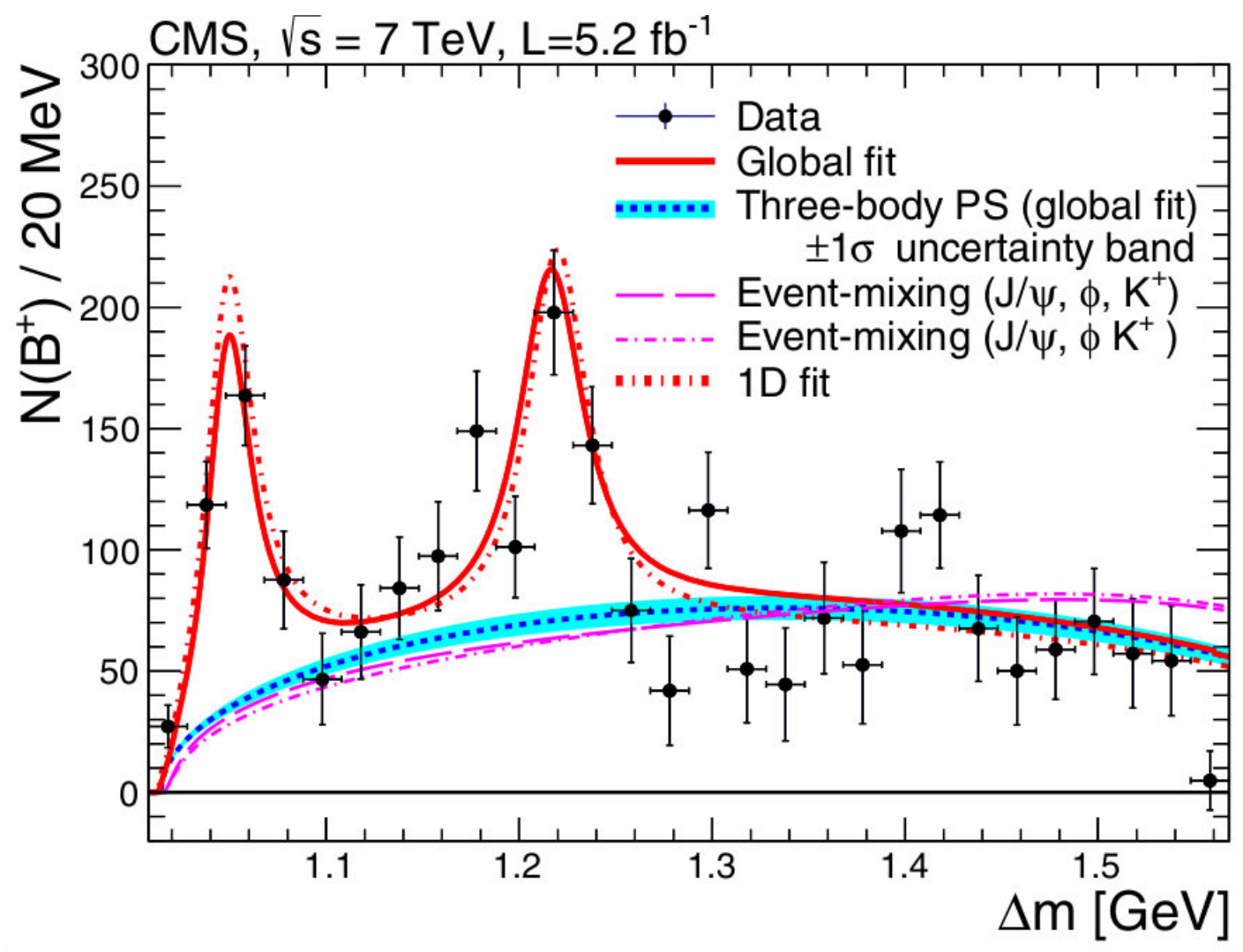

Figure 2. Number of $B^{+} \rightarrow J / \psi \phi K^{+}$candidates as a function of $\Delta m=m\left(\mu^{+} \mu^{-} K^{+} K^{-}\right)-m\left(\mu^{+} \mu^{-}\right)$. The solid curve is the global UML fit of the data, and the dotted curve is the background contribution assuming three-body phase space. The band is the $\pm 1 \sigma$ uncertainty range for the background obtained from the global fit. The dashed and dash-dotted curves are background curves obtained from two different event-mixing procedures and normalized to the number of three-body phase space background events. The short dashed curve is the 1D fit to the data.

Breit-Wigner function convolved with a Gaussian resolution function whose width is fixed to the value obtained from simulation. The mass resolution is about $1 \mathrm{MeV}$ at the threshold and smoothly increases with increasing $\Delta m$ : it is about $4 \mathrm{MeV}$ for $\Delta m \simeq 1.25 \mathrm{GeV}$. A $4.5 \mathrm{MeV}$ smearing is applied to the three-body phase-space profile to account for the width of the recostructed $\phi$ signal. The signal yields associated to the two structures are $355 \pm 46$ and $445 \pm 83$ respectively. On the same plot is also shown the result of a 2D simultaneous fit of both $B^{+}$invariant mass and $\Delta m$ distributions with implicit background subtraction and efficiency correction; the $\Delta m$ continuum shape has been also investigated with an event-mixing technique. There are clearly enhancements, with respect to the phase-space continuum: at the kinematical threshold and around $\Delta m \approx 1.2 \mathrm{GeV}$. The three-body phase-space background lies above the event-mixed background in the region of these two structures.

Several checks have been done to validate the robustness of the two structures. Not only each selection requirement has been varied but the whole analysis has been repeated with tighter selection criteria that lowered the combinatorial background level by a factor of ten while retaining $40 \%$ of the $B$ signal candidates. 


\section{$3.3 Y(4140)$ results}

Interpreting the two structures as $J / \psi \phi$ resonances with $S$-wave relativistic Breit-Wigner lineshapes laying over a residual three-body phase-space non-resonant component, the fitted mass of the first structure is $m=(4148.2 \pm 2.0$ (stat.) \pm 4.6 (syst.) $) \mathrm{MeV}$ whereas that of the second structure is $m=(4316.7 \pm 3.0$ (stat. $) \pm 7.3$ (syst.) $) \mathrm{MeV}$. The fitted width of the first structure is $\Gamma=\left(28_{-11}^{+15}\right.$ (stat. $) \pm$ 19 (syst.)) MeV. Preliminary systematic uncertainties' evaluation has been obtained by changing signal and background fit models, $\Delta m$ binning, mass resolution, efficiency correction and selection criteria. The first structure is observed with a significance exceeding $5 \sigma$; this is evaluated either by performing a likelihood ratio test and applying Wilks's theorem, and by performing toy-MC simulation to calculate a $p$-value for the background fluctuations alone to give rise to a signal as significant as that seen in the data. This observation is consistent with a previous evidence for a narrow structure near $J / \psi \phi$ threshold by the CDF Collaboration [12].

The background-subtracted $\phi K^{+}$invariant mass distribution shows an excess with respect to the pure phase-space profile in the $1.7-1.8 \mathrm{GeV}$ region where large resonances, such as $K_{2}(1770)$ and $K_{2}(1820)$, may appear. The reflections studies subsequently carried out suggest that the first structure is not correlated to eventual $\phi K^{+}$resonances.

\section{Search for the $X(5568)$ in the CMS data}

Recently the $\mathrm{D} \varnothing$ Collaboration claimed the evidence of a narrow state in the $B_{s}^{0} \pi^{ \pm}$invariant mass spectrum with mass $m=\left(5567 \pm 2.9_{-1.9}^{+0.9}\right) \mathrm{MeV}$ and $\Gamma=\left(21.9 \pm 6.4_{-2.5}^{+5.0}\right) \mathrm{MeV}$ [16], thus raising a lot of interest within the community that studies exotic hadrons [17] and triggered this search at several hadron collider experiments, including CMS [18] and LHCb [19]. The CMS search for resonancelike structures in the $B_{s}^{0} \pi^{ \pm}$invariant mass spectrum was performed using an integrated luminosity of $19.7 \mathrm{fb}^{-1}$ of pp collisions at $\sqrt{s}=8 \mathrm{TeV}$. The $B_{s}^{0}$ candidates are recostructed in the decay chain $B_{s}^{0} \rightarrow J / \psi \phi, J / \psi \rightarrow \mu^{+} \mu^{-}, \phi \rightarrow K^{+} K^{-}$. The $B_{s}^{0} \pi^{ \pm}$invariant mass distributions do not show any unexpected structures for different kinematic requirements imposed to the $\pi^{ \pm}, B_{s}^{0}$ and $B_{s}^{0} \pi^{ \pm}$candidates. An upper limit on the relative production of $X(5568)$ and $B_{s}$ multiplied by the branching fraction of the decay $X(5568) \rightarrow B_{s} \pi^{ \pm}$is estimated to be $3.9 \%$ at $95 \%$ CL in the most conservative case.
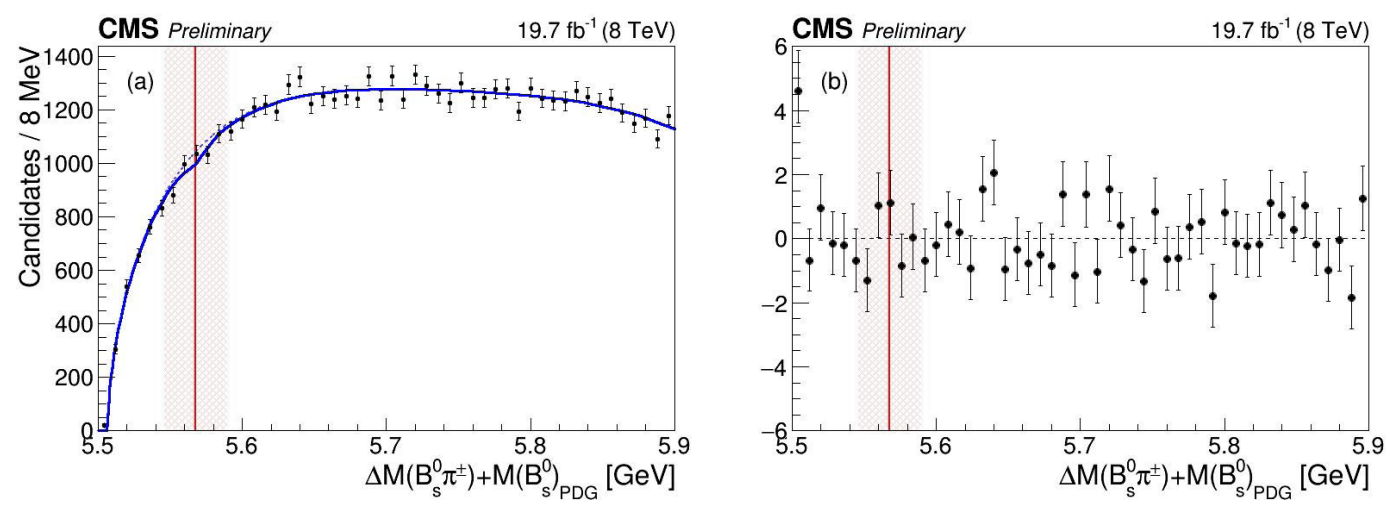

Figure 3. (left) Mass distribution of events in the $B_{s}$ signal region (black points with error bars) with fit results superimposed (blue line).(right) The pull distribution for (left): the (red) vertical band indicates the region of the $\mathrm{D} \varnothing$ observation around the mass of the claimed state. 


\section{Conclusions}

The $X(3872)$ production cross section is measured in $p p$ collisions at $\sqrt{s}=7 \mathrm{TeV}$ with data collected by the CMS experiment in 2011. The $X(3872)$ and $\psi(2 S)$ are observed using their decay into $J / \psi \pi^{+} \pi^{-}$. The ratio of their cross sections times branching fractions and the fraction of $X(3872)$ from $B$-hadron decays are measured. Neither of them shows any significant dependence on $p_{\mathrm{T}}$. Using these measurements, the prompt $X(3872)$ production cross section is obtained as a function of $p_{\mathrm{T}}$ for the first time. The available theoretical predictions significantly overestimate it while the dependence on $p_{\mathrm{T}}$ is reasonably described by the theory. The study of the dipion mass spectrum for $X(3872) \rightarrow J / \psi \pi^{+} \pi^{-}$ favours the presence of an intermediate $\rho^{0}$ state in the $X(3872)$ decay, thus confirming previous measurements by $\mathrm{CDF}$ and $\mathrm{LHCb}$.

The observation of the first structure in the $J / \psi \phi$ mass spectrum in $B$ hadron decays is consistent with a previous evidence by the CDF Collaboration. However the $\sqrt{s}=7 \mathrm{TeV}$ data do not provide an enough and pure sample to properly study the $J / \psi \phi$ system by performing an amplitude analysis of the 5-body decay mode to take into account the helicity configurations of the decay products.

The $B_{s}^{0} \pi^{ \pm}$invariant mass distributions do not show any unexpected structures for different kinematic requirements imposed to the $\pi^{ \pm}, B_{s}^{0}$ and $B_{s}^{0} \pi^{ \pm}$candidates. Hence an upper limit on the relative production of $X(5568)$ and $B_{s}$ multiplied by the branching fraction of the decay $X(5568) \rightarrow B_{s} \pi^{ \pm}$is estimated.

\section{References}

[1] Belle Collaboration, Phys. Rev. Lett. 91, 262001 (2003)

[2] N. Brambilla et al., Eur. Phys. J. C 71, 1534 (2011)

S. L. Olsen, Front. Phys. 10, 2, 121-154 (2015)

[3] CMS Collaboration, Phys. Lett. B 734, 261-281 (2014)

[4] CMS Collaboration, JINST 3, S08004 (2008)

[5] CMS Collaboration, JHEP 04,154 (2013)

[6] LHCb Collaboration, Phys. Rev. Lett. 110, 222001 (2013)

LHCb Collaboration, Phys. Rev. D 92, 011102 (2015)

[7] CMS Collaboration, JHEP 02, 011 (2012)

[8] P. Artoisenet and E. Braaten, Phys. Rev. D 81, 114018 (2010)

[9] CDF Collaboration, Phys. Rev. Lett. 96, 102002 (2006)

[10] Belle Collaboration, Phys. Rev. Lett. 94, 182002 (2005)

[11] Babar Collaboration, Phys. Rev. Lett. 101, 082001 (2008)

[12] CDF Collaboration, Phys. Rev. Lett. 102, 242002 (2009)

CDF Collaboration, arXiv:1101.6058, (2011)

[13] LHCb Collaboration, Phys. Rev. B 85, 091103 (2012)

[14] LHCb Collaboration, arXiv:1606.07898 (2016)

[15] M. Pivk and F. LeDiberder, Nucl. Instrum. Meth. A 555, 356 (2005)

[16] V. M. Abazov et al., Phys. Rev. Lett., 117(2):022003 (2016)

[17] T.J. Burns and E.S.Swanson, Phys. Lett. B 760, 627 (2016)

F.-K. Guo et al., Commun. Theor. Phys. 65(5), 593 (2016)

A.Ali et al., Phys. Rev. D 94, 034036 (2016)

[18] CMS Collaboration, CMS-PAS-BPH-16-002, https://cms-results.web.cern.ch/cms-results/ public-results/preliminary-results/BPH-16-002/ (2016)

[19] LHCb Collaboration, Phys. Rev. Lett. 117, 152003 (2016) 